\title{
Heterogeneization of alpha diimines nickel catalysts for the polymerization of ethylene and methylmethacrylate
}

\author{
Inês Matos, ${ }^{14}$ Auguste Fernandes, ${ }^{2}$ Rita Catalão, ${ }^{2}$ Ana M. Botelho do Rego, ${ }^{3}$ José $R$.
} Ascenso, ${ }^{1}$ Isabel F. Fonseca, ${ }^{4}$ Francisco Lemos, ${ }^{2}$ Maria M. Marques ${ }^{1 *}$

${ }^{1 *}$ Centro de Química Estrutural, Instituto Superior Técnico, Departamento de Engenharia Química, Av. Rovisco Pais, 1049-001 Lisboa, Portugal. E-mail: Maria.v.marques@ist.utl.pt

${ }^{2}$ IBB - Institute for Biotechnology and Bioengineering, Centre for Biological and Chemical Engineering, Instituto Superior Técnico, Av. Rovisco Pais, 1049-001 Lisboa, Portugal; e-mail: qflemos@alfa.ist.utl.pt

${ }^{3}$ Centro de Química Física Molecular and Institute of Nanoscience and Nanotechnology (IN), Instituto Superior Técnico, Universidade Técnica de Lisboa, Av. Rovisco Pais, 1049-001 Lisboa, Portugal; e-mail: amrego@ist.utl.pt

${ }^{4}$ REQUIMTE-CQFB, Departamento de Química, Faculdade de Ciências e Tecnologia, Universidade Nova de Lisboa, 2825-114 Caparica, Portugal; e-mail: iss@dq.fct.unl.pt.

(Received: 27 February, 2009; published: 27 December, 2010)

\begin{abstract}
In this paper we present two different techniques for the preparation of single site heterogeneous catalyst. The first method consists in the impregnation of a solution of the organometallic compound in MCM41. The second method intends to establish the in situ synthesis of the complex within the solid's pores by the reaction of the organic ligand with the metal cation previously introduced in the support. The direct deposition of the organometallic complex in the support resulted in an active catalyst which gives polyethylene with the same microstructure as the one obtained with the related homogeneous systems. The heterogeneous catalysts obtained by reaction of the ligand with the metal already present in the support showed a lower catalytic activity.
\end{abstract}

\section{Introduction}

Single site catalysts present a number of advantages. Among the most important ones is the ability to control the properties of the synthesized polymers, such as stereoregularity and polymer weight distribution [1]. Also, the homogeneous nature of these catalysts makes them an easier object of study when compared to heterogeneous catalysts which tend to possess a wide variety of different active sites [2].

However, in order to apply these catalysts at an industrial level it is important that they can be easily adapted for the use in the current facilities, which means that heterogeneous catalysts are preferred.

In this context much work has been developed towards the heterogeneization of single site catalysts [3], always seeking to maintain most of the homogeneous catalysts characteristics or if possible improve them, while ensuring the advantages of a heterogeneous catalyst. The main challenge is to guarantee that the 
heterogeneous catalyst shows a behavior similar to the homogeneous system while maintaining the same requirements as the traditional heterogeneous catalyst. As such this remains an area of active research interest.

Many methods have been used for the preparation of heterogeneous catalysts from molecular catalysts [4]. The majority of the heterogeneous single site catalysts are compounds activated by MAO supported in inorganic oxides. The most common support material is silica, but a variety of other materials like zeolites $Y$ and ZSM5, alumina, $\mathrm{MgCl}_{2}$ and more recently mesoporous material like $\mathrm{MCM} 41$ have been tested in these studies. Four main methods for the heterogeneization procedure can be identified:

I. Pre-treatment of the surface with MAO followed by impregnation of a solution of the precatalyst

II. Direct impregnation of the precatalyst in the support

III. Impregnation of a previously prepared solution of MAO/Precatalyst

IV. Covalent bonding of the precatalyst in the support by reaction with the functional groups present in the surface

The first method is among those that have been used for a longer time and it is still the most used one. It consists of contacting a solution of MAO, normally in toluene, with the support material, followed by careful washing. The modified support, after having been dried, is put in contact with a solution of the complex that is used as catalyst. Welborn and Takahashi applied this method for $\mathrm{Cp}_{2} \mathrm{ZrX}_{2}$ in silica and obtained an active catalyst for the polymerization of ethylene and copolymerization of others alpha olefins [5]. Some variations of this method, like using higher temperatures during the impregnation of $\mathrm{MAO}$, even performing this step under reflux conditions, seem to improve the performance of the final catalyst. Different temperatures in the complex solution impregnation also affect the final result. Soares and co-workers presented the in situ impregnation of the precatalyst into the modified silica inside the polymerization reactor, as an alternative for more efficient heterogeneous catalysts [6-8]. Chemical modification of the support prior to the impregnation of MAO has also been applied as a way to control the functional groups present on the surface of the support. Catalysts prepared with some modified silicas presented enhanced activity when compared to the ones prepared using the untreated surface $[9,10]$.

The second method corresponds to the direct impregnation of the complex in the support, and it is less common. Due to the interaction of the organometallic complex with the functional groups on the surface of the support, the calcination temperature of the silica is an important factor. Again chemically treated silicas were used in some studies and resulted in catalysts with higher catalytic activity and polymer with narrower molecular weight dispersion [11-13].

The third method consists in the preparation of a homogeneous solution of MAO and the complex and then contacting it with the support. This procedure reduces the amount of solvent used in the catalyst preparation, maximizes the formation of active sites since the activation occurs in homogeneous medium with less diffusion limitations, and it allows for the preparation of catalysts with low $\mathrm{Al} / \mathrm{M}$ ratio [14].

The most common method to establish a covalent bonding to the support is through reaction with the functional group present in the material. When using silica, these 
groups are usually $\mathrm{OH}$ but it is possible to produce different functional groups by adequate chemical treatment of the support. This method has been applied using two different procedures: the direct synthesis of the complex molecules inside the support [15], or the synthesis of a precursor ensuring that it has a reactive group in its structure capable of bonding to the surface groups [4]. The synthesis of the catalytic precursor may require a step by step construction [17]. One of the reasons pointed for the application of this method is to prevent leaching of the active compound; however Hlatky [16] questions the necessity for covalent bonding since leaching is not the only cause for bad particle morphology and other methods have proven to be able to prevent leaching. The other main advantage for this technique is that the structure of the complex remains intact and known.

In this paper we present the study of heterogeneous catalysts prepared by two different techniques (or methods) using two different support materials. The first method consisted in the direct impregnation of the organometallic compounds in the MCM41, used as the support. In the second method, the metal that constitutes the nucleus of the catalytic precursor was introduced in the support ( $\mathrm{NaY}$ ) by ion exchange after which the resulting material was contacted with a ligand solution in order to promote its coordination to the metal cations and thus obtaining the desired catalysts. The aim of this work was to develop new catalysts and to study the effect of the heterogenization procedure on the polymerization rate and on the properties of the polymer that is produced.

\section{Results and discussion}

\section{Catalysts characterization}

The compounds were prepared as described in the experimental part and were characterized by various techniques. The amount of nickel present in the catalysts prepared by the first method (method A) was determined by ICP. Compound $1 / \mathrm{MCM} 41$ has $0.18 \% \mathrm{Ni}$ whereas $2 / \mathrm{MCM} 41$ presents $0.07 \%$ of $\mathrm{Ni}$. This result allows us to establish the possible number of active centres for the polymerization of ethylene. It is clear from these results that, although it is possible to prepare a heterogeneous nickel -based catalyst by this method, the efficiency of impregnation procedure is not very high. Taking into account that a good dispersion of the metal on the support is an important feature for these heterogeneous catalysts, this low incorporation values may, nevertheless, represent an advantage.

The BET surface area, total pore volume and the amount of nickel present in the sample are presented in Table 1. After impregnation of the compounds in the MCM41 the values of surface area and total pore volume decrease. The loss of $20 \%$ in BET surface area is consistent with the presence of a complex in the pores of the support, probably due to the size of the organic ligand.

The catalysts prepared by the Method B (in situ synthesis of the catalyst precursor see experimental section) were characterized by XPS and by UV-Vis DRS spectrophotometry. The XPS analysis showed the presence of nitrogen in the samples of catalysts prepared over zeolite $Y, L 1 / N i / Y$ and $L 2 / N i / Y$, but no nitrogen could be detected in the samples prepared over MCM41. This result indicates that this second method is not adequate to prepare supported catalysts when the support used is MCM41. Lixiviation of the reaction product seems to occur since no evidence of the presence of the ligand could be detected in this case. 
Tab.1. Structural characterization.

\begin{tabular}{c|c|c|c|c}
\hline Catalyst & $\begin{array}{c}\text { Support } \\
\text { material }\end{array}$ & $\begin{array}{c}\text { BET surface area } \\
\mathbf{~}^{2} / \mathbf{g}\end{array}$ & $\begin{array}{c}\text { Total pore } \\
\text { volume } \\
\mathbf{c m}^{3} / \mathbf{g}\end{array}$ & $\begin{array}{c}\text { Amount of } \mathbf{~ N i} \\
\text { ICP } \%\end{array}$ \\
\hline MCM41 & MCM41 & 1249 & 1.087 & \\
NaY & Zeolite NaY & 662 & 0.34 & 0.18 \\
1/MCM41 & MCM41 & 966 & 0.93 & 0.07 \\
$2 / \mathrm{MCM} 41$ & MCM41 & 1022 & 0.99 & \\
$\mathrm{~L} 1 / \mathrm{NiY}$ a) & Zeolite NaY & 495 & 0.23 & \\
$\mathrm{~L} 2 / \mathrm{NiY}$ a) & Zeolite NaY & 414 & 0.19 & \\
\hline
\end{tabular}

The UV-Vis DRS analyses of the sample $\mathrm{L} 1 / \mathrm{Ni} / \mathrm{Y}$, the starting $\mathrm{Ni} / \mathrm{Y}$ and the organic ligand were carried out in order to determine if the ligand had established any interaction with the metal present in the zeolite. UV-Vis DRS has been used to study different solid compounds and has proven to be able to provide valuable information $[18,19]$. Results can be found in the literature where this technique was used to analyse nickel catalysts supported on zeolite or silica and where valuable information was obtained since the UV-Vis DRS spectrum reflects the metal coordination state [20].

The spectrum of the dry Ni/Y is presented in Figure 1 . It seems that there is only $\mathrm{Ni}^{2+}$ present in this sample. It is possible to identify four different bands, at $455,486,571$ and $612 \mathrm{~nm}$, which according to Che and Lepetit [21] correspond to a tetrahedral distorted geometry of $\mathrm{Ni}^{2+}$.

The bands of the spectrum of the ligand are observed at different values of wavelength, 315 and $390 \mathrm{~nm}$, as illustrated in Figure 2.

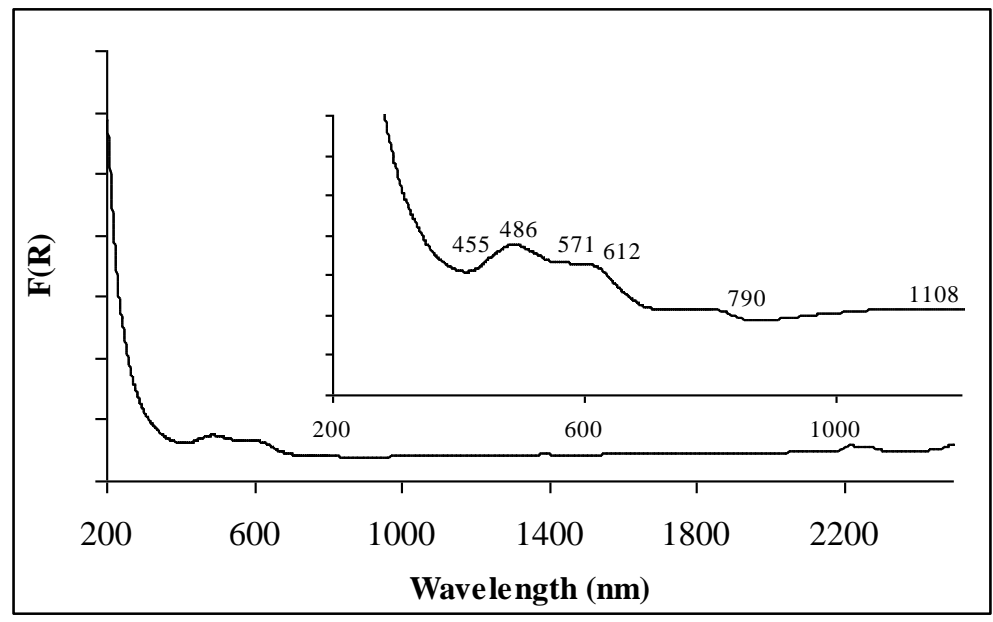

Fig. 1. UV-Vis DRS spectrum of $\mathrm{Ni} / \mathrm{Y}$, reflectance mode.

When compared to the previous spectra, the L1/NiY spectra (Figure 3 ) seem to show bands corresponding to the ligand at $325 \mathrm{~nm}$ and $406 \mathrm{~nm}$ which appear at slightly higher values compared to the ligand in solution due to the presence of the support and to the interaction with the metal cation. The band at $478 \mathrm{~nm}$ may suggest the presence of $\mathrm{Ni}^{2+}$ in square planar geometry [22]. Since these alfa diimine nickel complexes in solution do present square planar geometry geometry, which is also 
obtained when molecular modelling calculations are performed [23], this result indicates that some coordination of the ligand to the metal has occurred. This spectrum of $\mathrm{L} 1 / \mathrm{NiY}$ does not exclude the presence of $\mathrm{Ni}^{2+}$ in tetrahedrical geometry but it suggests that part of the nickel has been coordinated to the ligand thus gaining a different geometry.

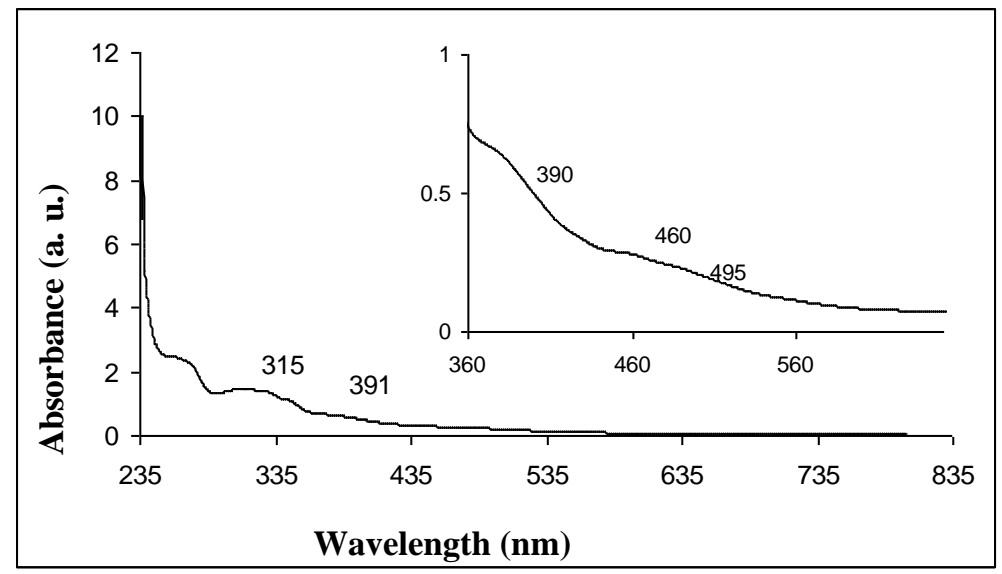

Fig. 2. UV-Vis DRS spectrum of compound L1 in solution.

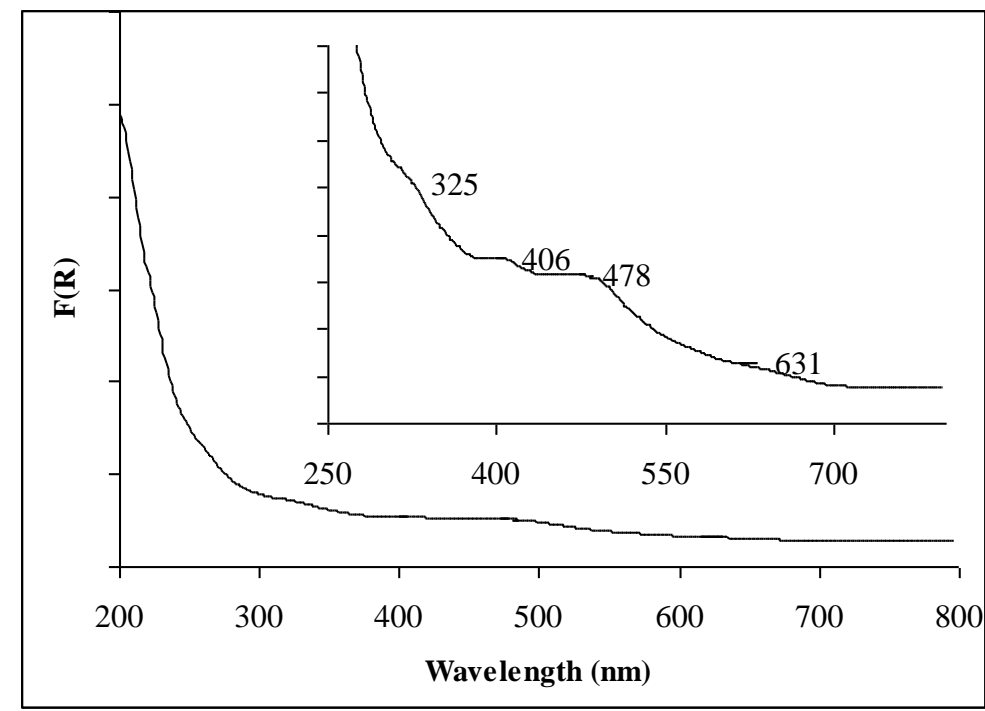

Fig. 3. UV-Vis DRS spectrum of compound L1/NiY, reflectance mode.

The TGA analysis of this sample showed that the organic ligand content in the catalyst corresponded to $4 \%$ of the total mass sample, which means that $15 \%$ of the nickel present may have reacted with the ligand.

\section{Ethylene polymerization}

The resulting catalysts were evaluated in the polymerization of ethylene under different experimental conditions. Table 2 shows the catalytic results obtained with the catalysts prepared by Method A (direct impregnation), 1/MCM41 and 2/MCM41 when using different cocatalysts. 
For both compounds the best cocatalysts are DEAC and MAO. The difference of activity between these two cocatalysts is not meaningful whatever the catalyst is. With the other cocatalysts studied, TMA and TIBA, it is possible to obtain polymer but the activity is very low. These results are similar to those obtained with these catalyst precursors when studied in homogeneous phase, a fact that reinforces the similarity between the homogenous and the corresponding heterogenized catalysts.

The activity value for the heterogeneous system remains high, although suffering a reduction of one order of magnitude when compared to the homogeneous systems. However these values are similar to some reported in the literature for alpha diimine nickel complexes [24-26].

Tab. 2. Homopolymerization of Ethylene by 1/MCM41 and 2/MCM41: cocatalyst effect.

\begin{tabular}{c|c|c|c|c|c}
\hline Catalyst & Run & Cocatalyst & [Al]/[Ni] & $\begin{array}{c}\text { m } \\
\text { g pol }\end{array}$ & $\begin{array}{c}\text { Activity } \\
\text { KgPol/(molNi.h.bar) }\end{array}$ \\
\hline & PSNi 16 & TMA & 7547 & 0.0571 & $4.00 \times 10^{+2}$ \\
1/MCM41 $^{\text {a) }}{ }^{2}$ & PSNi 17 & TIBA & 7547 & 0.0422 & $2.95 \times 10^{+2}$ \\
& PSNi 7 & DEAC & 7575 & 0.3027 & $20.07 \times 10^{+2}$ \\
& PSNi 9 & MAO & 8023 & 0.2274 & $15.60 \times 10^{+2}$ \\
\hline & PSNi 21 & TMA & 18884 & 0.0118 & $2.05 \times 10^{+2}$ \\
$2 /$ MCM41 $^{\text {b) }}$ & PSNi 22 & TIBA & 18884 & 0.023 & $3.96 \times 10^{+2}$ \\
& PSNi 12 & DEAC & 18928 & 0.152 & $26.00 \times 10^{+2}$ \\
& PSNi 14 & MAO & 19044 & 0.1175 & $20.10 \times 10^{+2}$ \\
\hline
\end{tabular}

Experimental conditions: $V=50 \mathrm{ml}$ Toluene; $\mathrm{T}=25^{\circ} \mathrm{C} ; \mathrm{t}_{\mathrm{p}}=30 \mathrm{~min} ; \mathrm{P}$ Ethylene $=1 \mathrm{bar} ;[\mathrm{Nii}]=5.81 \times 10^{-6} \mathrm{M}$; $\left.\mathrm{m}_{\text {catalyst in reactor }}=9.5 \times 10^{-3} \mathrm{~g} ; \mathrm{b}\right)[\mathrm{Ni}]=2.32 \times 10^{-6} \mathrm{M} ; \mathrm{m}_{\text {catalyst in reactor }}=9.8 \times 10^{-3} \mathrm{~g}$

The effect of the amount of cocatalyst in the reaction medium is presented in Table 3. For system 1/MCM41/MAO the catalytic activity seems to increase steadily with the $\mathrm{Al} / \mathrm{Ni}$ ratio, in the experimental conditions studied. For system 2/MCM41/MAO it was possible to study higher ratios. There is an increase of the activity with the amount of aluminium until a maximum value of activity which is reached at an $\mathrm{Al} / \mathrm{Ni}$ of 60000 . For higher ratio values a decrease in activity is observed. Similar study with the catalytic system 2/MCM41/DEAC revealed that the behaviour is analogous but the maximum activity is reached at lower values of $\mathrm{Al} / \mathrm{Ni}$ which may represent an advantage of DEAC over MAO either for environmental reasons or economical ones.

The effect of temperature was studied for both catalysts. System 1/MCM41/MAO shows an increase in thermal stability compared to the homogeneous system, presenting a maximum of activity at $50^{\circ} \mathrm{C}$. This is a very positive result suggesting that the heterogenization grants a more stable catalyst which deactivates less with the increase of the temperature. However system 2/MCM41/MAO did not reveal any significant improvement of the activity in the same range of temperatures.

The polymer obtained in these reactions was analysed by ${ }^{1} \mathrm{H}$ and ${ }^{13} \mathrm{C} N M R$, and by TG/DSC. The polymer produced is branched, as it is clear by the NMR spectra. Figure 4 represents the spectra of polymer obtained in run KSNi1, and in another run under similar experimental conditions with the corresponding homogeneous system (top spectrum). It is possible to conclude that the polymers are very similar, which indicates that the support does not interfere with the microstructure of the polyethylene. Similar observations were made for catalyst 2/MCM41. 
Tab. 3. Homopolymerization of Ethylene by $1 / \mathrm{MCM} 41$ and $2 / \mathrm{MCM} 41$ : effect of $\mathrm{Al} / \mathrm{Ni}$ ratio and temperature.

\begin{tabular}{|c|c|c|c|c|c|c|}
\hline Catalyst & Run & Cocatalyst & {$[\mathrm{Al}] /[\mathrm{Ni}]$} & $\begin{array}{l}\mathrm{T} \\
{ }^{\circ} \mathrm{C}\end{array}$ & m pol & $\begin{array}{c}\text { Activity } \\
\text { kgPol/(molNi.h.bar) }\end{array}$ \\
\hline $1 / \mathrm{MCM}_{4} 1^{\mathrm{a})}$ & $\begin{array}{l}\text { PSNi } 20 \\
\text { KSNi } 1 \\
\text { PSNi } 18 \\
\text { PSNi } 19 \\
\text { KSNi15 } \\
\text { KSNi14 }\end{array}$ & MAO & $\begin{array}{l}3997 \\
16047 \\
23980 \\
31974 \\
16047 \\
16047\end{array}$ & $\begin{array}{l}0 \\
50\end{array}$ & $\begin{array}{l}0.3361 \\
0.4336 \\
0.622 \\
0.7715 \\
0.1951 \\
0.4099\end{array}$ & $\begin{array}{l}2.35 \times 10^{+3} \\
2.92 \times 10^{+3} \\
4.36 \times 10^{+3} \\
5.40 \times 10^{+3} \\
1.12 \times 10^{+3} \\
3.64 \times 10^{+3}\end{array}$ \\
\hline $2 / M C M 41^{b)}$ & $\begin{array}{l}\text { PSNi } 25 \\
\text { PSNi } 14 \\
\text { KSNi } 5 \\
\text { PSNi } 23 \\
\text { PSNi } 24 \\
\text { KSNi } 8 \\
\text { KSNi } 16\end{array}$ & MAO & $\begin{array}{l}10000 \\
19044 \\
40093 \\
60000 \\
80001 \\
40093 \\
40093\end{array}$ & $\begin{array}{l}50 \\
0\end{array}$ & $\begin{array}{c}0.1418 \\
0.1175 \\
0.1957 \\
0.2108 \\
0.1388 \\
0.1046 \\
0.02\end{array}$ & $\begin{array}{l}2.47 \times 10^{+3} \\
2.01 \times 10^{+3} \\
3.36 \times 10^{+3} \\
3.64 \times 10^{+3} \\
2.41 \times 10^{+3} \\
1.80 \times 10^{+3} \\
3.48 \times 10^{+2}\end{array}$ \\
\hline 2/MCM41 & $\begin{array}{l}\text { PSNi } 11 \\
\text { PSNi } 12 \\
\text { PSNi } 13\end{array}$ & DEAC & $\begin{array}{c}9464 \\
18928 \\
28392\end{array}$ & 25 & $\begin{array}{c}0.1478 \\
0.152 \\
0.1187\end{array}$ & $\begin{array}{l}2.53 \times 10^{+3} \\
2.60 \times 10^{+3} \\
2.03 \times 10^{+3}\end{array}$ \\
\hline
\end{tabular}

Experimental conditions: a) $[\mathrm{Ni}]=5.81 \times 10^{-6} \mathrm{M} ; \mathrm{m}$ catalyst in reactor $=9.5 \times 10^{-3} \mathrm{~g} ; \mathrm{V}=50 \mathrm{ml}$ Toluene; $\mathrm{T}=25^{\circ} \mathrm{C}$; $t_{p}=30 \mathrm{~min} ; P_{\text {Ethylene }}=1$ bar; b) $[\mathrm{Ni}]=2.32 \times 10^{-6} \mathrm{M} ; \mathrm{m}$ catalyst in reactor $=9.8 \times 10^{-3} \mathrm{~g} ; \mathrm{V}=50 \mathrm{ml}$ Toluene; $t_{p}=30 \mathrm{~min}$; $P_{\text {abs }}$ Ethylene $=2$ bar.

The resonance assignments of branched polyethylene were done as described by Galland et al [27]. These polymers present a total branching of $6.4 \%$ for system $1 / \mathrm{MCM} 41 / \mathrm{MAO}$ and 2,4\% for 2/MCM41/MAO, values which are similar to the ones obtained with the homogeneous systems. These are, however, less branched polymers than those obtained with other alpha-diimine nickel catalysts which are reported in literature as having more than $10 \%$ of total branching $[26,28]$. In the polymer samples prepared with our systems only isolated methyl branches could be detected. The resonance at 19.9 ppm corresponds to the methyl carbon, that at 27.4 
ppm correspond to the carbon in the $\beta$ position, at $33.2 \mathrm{ppm}$ is the tertiary carbon and at $37.5 \mathrm{ppm}$ the carbon in $\alpha$ position to the branch.

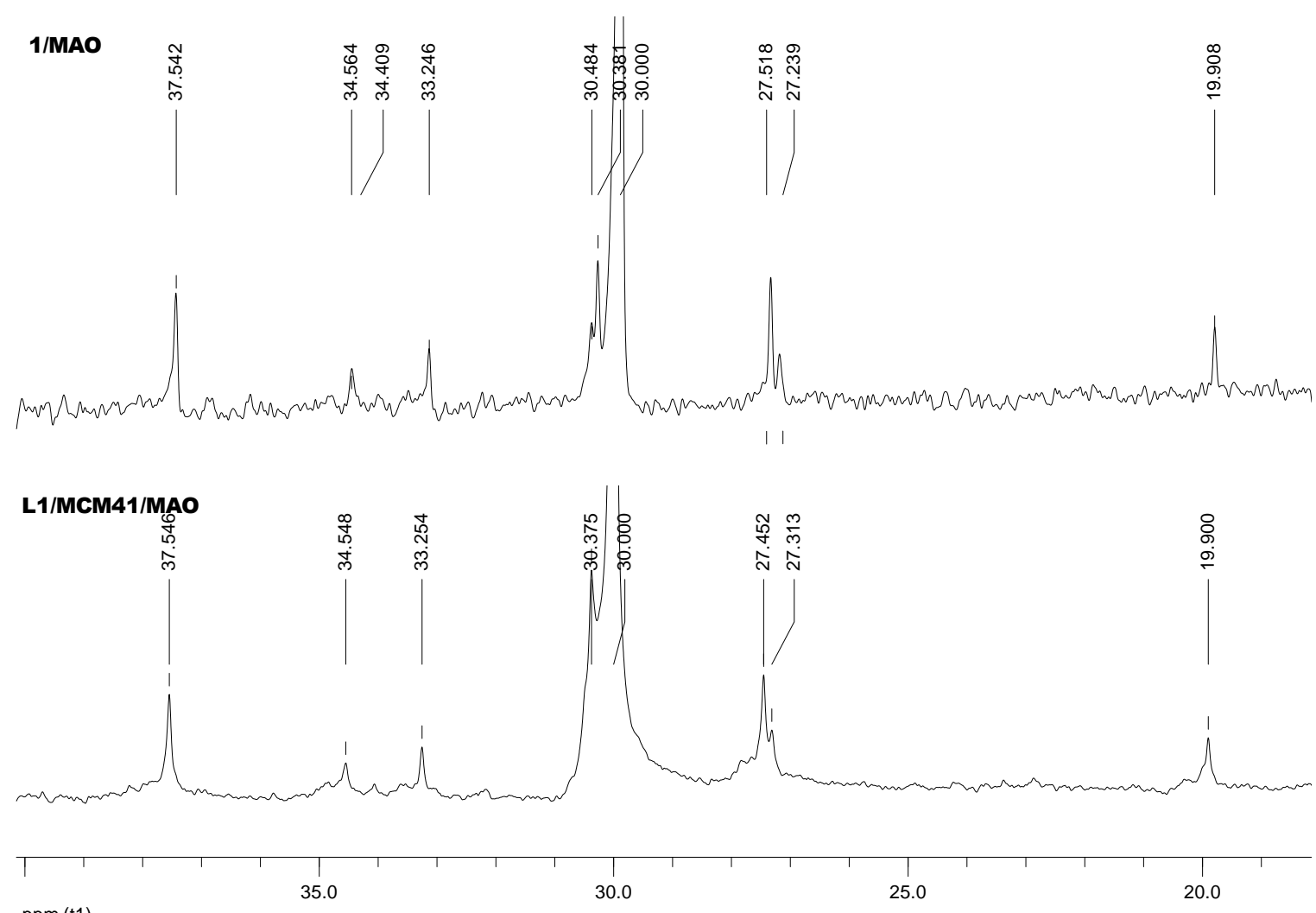

Fig. 4. ${ }^{13} \mathrm{C}$ NMR spectra of polyethylene obtained in similar experimental conditions with the homogeneous system (top spectrum) and with the heterogeneous system (lower spectrum).

Table 4 shows the thermal properties of the polymers and the thermogravimetric results. $T_{\text {ons }}$ and $T_{\max }$ represent the onset and maximum temperatures of the melting peaks, respectively, and $\Delta \mathrm{H}_{\mathrm{f}}$ is the enthalpy of fusion. These results show that the melting point temperature of the polymers obtained with these new heterogeneous systems does not vary much from those obtained with the homogeneous systems, although catalyst $1 / \mathrm{MCM} 41$ produces polymers with slightly lower melting points. On the other hand the enthalpy of fusion shows much lower values. This fact may not be related with a decrease in crystallinity but rather be due to the fact the polymer is contaminated by the inorganic support, as reported previously in the literature [29]. These results support the conclusion that the microstructures of the polymers are similar.

The differences observed in the thermal properties of the polymers obtained with the same catalyst, but at a different temperature, are of some significance for both systems, but again this effect is very similar to that observed in the case of the polymers obtained from homogeneous systems.

The catalysts obtained by the Method B (in situ synthesis), prepared by reaction of the ligand with $\mathrm{Ni} / \mathrm{Y}$, were also studied in the polymerization of ethylene. The compounds L1/NiY, L2/NiY and L3/NiY are less active than the ones prepared by the previous method. However there is an increase in activity when compared to the 
catalyst $\mathrm{Ni} / Y$ prepared by ion exchange and which was used as starting material for the new compounds, and was also studied in ethylene polymerization.

Tab. 4. Thermal properties of polyethylene by 1/MCM41 and 2/MCM41.

\begin{tabular}{|c|c|c|c|c|c|c|}
\hline \multirow[t]{2}{*}{ Run } & \multirow[t]{2}{*}{ Catalyst } & \multirow{2}{*}{$\begin{array}{l}T_{p} \\
{ }^{\circ} \mathrm{C}\end{array}$} & \multirow{2}{*}{$\begin{array}{c}\% \\
\text { Inorganic matter }\end{array}$} & \multicolumn{3}{|c|}{ first heating run } \\
\hline & & & & $\mathrm{T}_{\text {ons }} /{ }^{\circ} \mathrm{C}$ & $\mathrm{T}_{\max } /{ }^{\circ} \mathrm{C}$ & $\Delta \mathrm{H} / \mathrm{Jg}^{-1}$ \\
\hline KSNi 1 & & 25 & 9.2 & 76.0 & 91.7 & 47.3 \\
\hline KSNi 14 & 1/MCM41 & 50 & 2.3 & 40.4 & 46.2 & 0.68 \\
\hline LNi19 & & 18 & 0 & 89.5 & 109.9 & 108.8 \\
\hline KSNi 5 & & 25 & 0.5 & 114.3 & 127.4 & 134.0 \\
\hline KSNi 8 & 2/MCM41 & 50 & 8.6 & 77.7 & 89.0 & 54.7 \\
\hline KNiY3 & & 25 & 0 & 115.6 & 127.4 & 140.5 \\
\hline
\end{tabular}

Tables 5 to 7 show the catalytic results for the three catalysts in different experimental conditions.

Tab. 5. Homopolymerization of Ethylene by L1/NiY, L2/NiY, L3/NiY: effect of the cocatalyst.

\begin{tabular}{|c|c|c|c|c|c|}
\hline Catalyst & Run & Cocatalyst & {$[\mathrm{Al}] /[\mathrm{Ni}]$} & $\underset{g}{\mathrm{~m} \text { pol }}$ & $\begin{array}{c}\text { Activity } \\
\mathrm{kgPol} /(\text { molNi.h.bar) }\end{array}$ \\
\hline $\mathrm{Ni} / \mathrm{Y}^{\mathrm{b})}$ & KNi5 & DEAC & 300 & 0.0161 & 0.77 \\
\hline \multirow{4}{*}{$\mathrm{L}_{1} / \mathrm{NiY}^{\mathrm{a}}$} & PSNi 26 & TMA & 411 & traces & - \\
\hline & PSNi 27 & TIBA & 411 & traces & - \\
\hline & PSNi 29 & DEAC & 411 & 0.0236 & 8.50 \\
\hline & PSNi 28 & MAO & 411 & 0.0169 & 6.12 \\
\hline \multirow{4}{*}{ L2/NiY a) } & PSNi 46 & TMA & 411 & traces & - \\
\hline & PSNi 47 & TIBA & 411 & traces & - \\
\hline & PSNi 48 & DEAC & 411 & 0.0227 & 8.24 \\
\hline & PSNi 49 & MAO & 411 & traces & 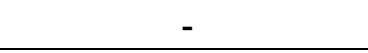 \\
\hline \multirow{4}{*}{$\mathrm{L} / \mathrm{NiY}^{\mathrm{a}}$} & PSNi 61 & TMA & 411 & 0.0084 & 3.02 \\
\hline & PSNi 62 & TIBA & 411 & traces & - \\
\hline & PSNi 63 & DEAC & 411 & 0.0086 & 3.13 \\
\hline & PSNi 64 & MAO & 411 & traces & 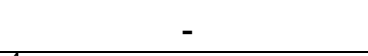 \\
\hline
\end{tabular}

Experimental conditions: $\mathrm{T}=25^{\circ} \mathrm{C} ; \mathrm{V}=50 \mathrm{ml}$ toluene ${ }^{\text {a) }}[\mathrm{Ni}]=1.07 \times 10^{-4} \mathrm{M} ; \mathrm{m}$ catalyst in reactor $=1.85 \times 10^{-2} \mathrm{~g}$; $t_{p}=30 \mathrm{~min} ; P_{\text {Ethylene }}=1$ bar; ${ }^{b}[\mathrm{Ni}]=5.78 \times 10^{-4} \mathrm{M} ; \mathrm{t}_{\mathrm{p}}=60 \mathrm{~min} ; \mathrm{P}_{\text {Ethylene }}=0.7 \mathrm{bar}$

The values of activity per active site were determined considering the total amount of nickel in the sample, even though more than one kind of active centre may be present since not all the metal centres have reacted with the organic ligand, as it was seen by the characterization of the catalysts. The weight of recovered polymer from the reaction presented in the tables already accounts for the necessary correction regarding the inorganic matter trapped within the polymer. So, the amount of inorganic matter detected by TGA was subtracted from to the total amount of product recovered from the reaction.

The results show that the three catalyst samples studied present an increase in the activity of over ten times more than that observed for the NiY system with no ligand addition. The best cocatalyst in the polymerization of ethylene is DEAC, but MAO is also able to activate these samples, contrary to what happened with the NiY systems since only traces of polymer could be obtained with NiY/MAO. However, a higher 
concentration of MAO is required and even so the activity is lower than that observed for the DEAC systems. For the different compounds, the maximum of activity is observed at an $\mathrm{Al} / \mathrm{Ni}$ ratio between 820 and 1300 when DEAC is used as cocatalyst and between 1300 and 1700 when MAO is present in the reaction. The best reaction temperature is $25^{\circ} \mathrm{C}$ for the three catalysts.

The differences in the organic ligand structure resulted in few differences in the catalytic behaviour of the final catalyst. One could expect ligand L3 to have better access to the metal in the support due to its smaller dimensions, but there is no evidence to support this assertion, either in the characterization of the catalyst or in the catalytic results.

Tab. 6. Homopolymerization of Ethylene by L1/NiY, L2/NiY, L3/NiY: effect of Al/Ni.

\begin{tabular}{c|c|c|c|c|c}
\hline Catalyst & Run & Cocatalyst & {$[$ Al]/[Ni] } & $\begin{array}{c}\text { m } \\
\text { g pol }\end{array}$ & $\begin{array}{c}\text { Activity } \\
\text { kgPol/(molNi.h.bar) }\end{array}$ \\
\hline \multirow{3}{*}{ L1/NiY } & PSNi 31 & & 205 & 0.0192 & 7.18 \\
& PSNi 29 & DEAC & 411 & 0.0236 & 8.82 \\
& PSNi 30 & & 822 & 0.0255 & 9.53 \\
\hline \multirow{5}{*}{ L2/NiY } & PSNi 48 & & 412 & 0.0227 & 8.48 \\
& PSNi 56 & & 206 & 0.0220 & 8.23 \\
& PSNi 57 & DEAC & 824 & 0.0314 & 11.7 \\
& PSNi 58 & & 1236 & 0.0254 & 9.48 \\
& PSNi 59 & & 1648 & 0.0313 & 11.7 \\
\hline \multirow{5}{*}{ L3/NiY } & PSNi 71 & & 205 & 0.0159 & 5.94 \\
& PSNi 63 & & 411 & 0.0086 & 3.21 \\
& PSNi 72 & \multirow{2}{*}{ DEAC } & 822 & 0.0160 & 5.98 \\
& PSNi 73 & & 1235 & 0.0240 & 8.97 \\
& PSNi 74 & & 1647 & 0.0109 & 4.07 \\
\hline
\end{tabular}

Experimental conditions: $[\mathrm{Ni}]=1,07 \times 10^{-4} \mathrm{M} ; \mathrm{m}_{\text {catalyst in reactor }}=1,85 \times 10^{-2} \mathrm{~g} ; \mathrm{V}=50 \mathrm{ml}$ toluene; $\mathrm{t}_{\mathrm{p}}=30 \mathrm{~min}$; $\mathrm{P}_{\text {Ethylene }}=1$ bar; $\mathrm{T}=25^{\circ} \mathrm{C}$

Tab. 7. Homopolymerization of Ethylene by L1/NiY, L2/NiY, L3/NiY: effect of temperature.

\begin{tabular}{|c|c|c|c|c|c|c|}
\hline Catalyst & Run & Cocatalyst & ${ }^{\mathrm{T}}$ & {$[\mathrm{Al}] /[\mathrm{Ni}]$} & $\underset{g}{\text { m pol }}$ & $\begin{array}{c}\text { Activity } \\
\mathrm{kgPol} /(\text { molNi.h.bar) }\end{array}$ \\
\hline \multirow{3}{*}{ L1/NiY } & PSNi 20a) & \multirow{3}{*}{ MAO } & 0 & 1292 & 0.0139 & 5.20 \\
\hline & PSNi 33 & & 25 & 1307 & 0.0217 & 8.11 \\
\hline & PSNi 19a) & & 50 & 1292 & 0.0059 & 2.21 \\
\hline \multirow{3}{*}{ L2/NiY } & PSNi 60 & \multirow{3}{*}{ MAO } & 0 & 872 & traces & - \\
\hline & PSNi 52 & & 25 & 872 & 0.0053 & 1.96 \\
\hline & PSNi 50 & & 50 & 872 & traces & - \\
\hline \multirow{3}{*}{ L3/NiY } & PSNi 70 & \multirow{3}{*}{ MAO } & 0 & 1292 & traces & - \\
\hline & PSNi 68 & & 25 & 1307 & 0.0095 & 3.55 \\
\hline & PSNi 65 & & 50 & 1292 & 0.0087 & 3.25 \\
\hline
\end{tabular}

Experimental conditions: $[\mathrm{Ni}]=1.07 \times 10^{-4} \mathrm{M} ; \mathrm{m}_{\text {catalyst in reactor }}=1.85 \times 10^{-2} \mathrm{~g} ; \mathrm{V}=50 \mathrm{ml}$ toluene; $\mathrm{t}_{\mathrm{p}}=30 \mathrm{~min}$; $P_{\text {Etylene }}=1$ bar; $\mathrm{T}=25^{\circ} \mathrm{C}$. ${ }^{\mathrm{a})}[\mathrm{Ni}]=2.02 \times 10^{-4} \mathrm{M}$

Some of the polymers obtained were characterized by ${ }^{1} \mathrm{H},{ }^{13} \mathrm{C}$ RMN and DSC. Again the polymer that is obtained is a branched polyethylene, as it is characteristic of the $\mathrm{Ni}$-based catalysts and, just like in the former samples, only isolated methyl branches can be detected in the ${ }^{13} \mathrm{C}$ spectra. The DSC results show that the polymers exhibit 
melting point temperatures similar to those observed for the polymers obtained with the homogeneous systems 1/MAO e 2/MAO.

Tab. 8. Thermal properties of polyethylene prepared by $L 1 / N i Y$ and $L 2 / N i Y$ and L3/NiY.

\begin{tabular}{ccccc}
\hline Run & Catalyst & \multicolumn{3}{c}{$\mathbf{1}^{\text {st }}$ Eating run } \\
\cline { 3 - 5 } & & $\mathbf{T}_{\text {onset }}{ }^{\circ} \mathbf{C}$ & $\mathbf{T}_{\max } /{ }^{\circ} \mathbf{C}$ & $\Delta \mathbf{H} / \mathbf{J g}^{-1}$ \\
\hline PSNi 30 & L1/NiY & 85.4 & 90.9 & 14.4 \\
PSNi 57 & L2/NiY & 117.7 & 129.1 & 90.5 \\
PSNi 73 & L3/NiY & 116.5 & 127.9 & 154.4 \\
\hline
\end{tabular}

\section{Polymerization of methyl methacrylate}

The homopolymerization of methyl methacrylate was tested in the presence of the homogeneous catalytic systems $1 / \mathrm{MAO}, 2 / \mathrm{MAO}$ and of the heterogeneous systems 1/MCM41/MAO and 2/MCM41/MAO. Table 9 presents the results obtained in this study.

The reactions were carried out in the absence of solvent and no other protective agent was added.

The polymerization of methyl methacrylate with high stereoregularity has been reported in the literature by Collins and Graham [30,31] using metalocene catalysts. Inukai [32] reported the synthesis of atactic polymer in the presence of $\mathrm{Ni}(\mathrm{acac}) 2 / \mathrm{MAO}$, and Woo [33] used Brookhart type catalyst in the homopolymerization of this monomer to obtain polymers with a good degree of syndiotacticity.

The results show that these catalytic systems are also able to polymerize MMA with some degree of syndiotacticity.

Tab. 9. Homo and co - polymerization of methyl metacrylate.

\begin{tabular}{|c|c|c|c|c|c|c|c|}
\hline Run & Catalyst & $\begin{array}{l}\text { E } \\
\text { bar }\end{array}$ & $\begin{array}{l}\mathrm{T} \\
{ }^{\circ} \mathrm{C}\end{array}$ & $\begin{array}{l}\mathbf{T} \\
\mathbf{h}\end{array}$ & $\underset{\text { g pol }}{\mathbf{m}}$ & $\begin{array}{c}\text { Yield } \\
\text { gPol/gMon } \\
(\%)\end{array}$ & $\begin{array}{c}\% \\
\text { MMA }\end{array}$ \\
\hline LNi 158 & & - & -20 & 7 & 0.5647 & 6.03 & \\
\hline LNi 168 & & - & 0 & 7 & 1.571 & 16.7 & \\
\hline LNi 153 & 1/MAO & - & 30 & 24 & 1.2345 & 13.19 & \\
\hline LNi 160 & & 2 & 0 & 7 & 1.0768 & & 20 \\
\hline LNi 155 & & 1 & 30 & 24 & 1.2892 & & 60 \\
\hline LNi 166 & & 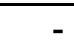 & 0 & 7 & 0.1004 & 1.1 & \\
\hline LNi 164 & (MA & - & 30 & 24 & 0.2507 & 2.68 & \\
\hline LNi 167 & & 2 & 0 & 7 & 0.0983 & & 60 \\
\hline LNi 165 & & 2 & 30 & 24 & 0.4115 & & 69 \\
\hline PSNi 41 & 1/MCM41 & - & 30 & 24 & 0.3826 & 4.1 & \\
\hline PSNi 43 & $/ \mathrm{MAO}^{\mathrm{b})}$ & - & 0 & 7 & 0.2280 & 2.4 & \\
\hline PSNi 42 & 2/MCM41 & - & 30 & 24 & 0.399 & 4.24 & \\
\hline PSNi 45 & $/ \mathrm{MAO}^{\mathrm{c})}$ & - & 0 & 7 & 0.1640 & 1.75 & \\
\hline
\end{tabular}

Experimental conditions: a) $\mathrm{Ni}=1.39 \times 10^{-5} \mathrm{~mol}$ in reactor; $\mathrm{Al} / \mathrm{Ni}=500,10 \mathrm{ml}$ of MMA. b) $\mathrm{Ni}=3.06 \times 10^{-6}$ $\mathrm{mol} ; \mathrm{Al} / \mathrm{Ni}=1906 ; \mathrm{c}) \mathrm{Ni}=1.18 \times 10^{-6} \mathrm{~mol} ; \mathrm{Al} / \mathrm{Ni}=4924$ 
System $1 /$ MAO is more active in the polymerization of MMA than system 2/MAO. The yields observed for system 1 are slightly lower than those reported by Woo [33] who mentions a yield of $18.99 \%$ for a similar $\alpha$-diimine Nickel catalyst at $30^{\circ} \mathrm{C}$. The best reaction temperature is $30^{\circ} \mathrm{C}$ for the majority of the catalysts studied However, catalyst 1 shows a slightly higher conversion at $0^{\circ} \mathrm{C}$ than at $30^{\circ} \mathrm{C}$. A similar effect of the temperature had also been observed by Woo [33] who reports a conversion of $21.9 \%$ at $-10{ }^{\circ} \mathrm{C}$ while at $30{ }^{\circ} \mathrm{C}$ this value is only $18.99 \%$. These apparently unexpected results come from the fact that in this type of reactions where both steps, coordination and insertion reaction are important, the temperature has opposite effects in each step. The increase of the temperature favours the dissociation step thus decreasing the concentration of the coordinated species but it increases the insertion rate.

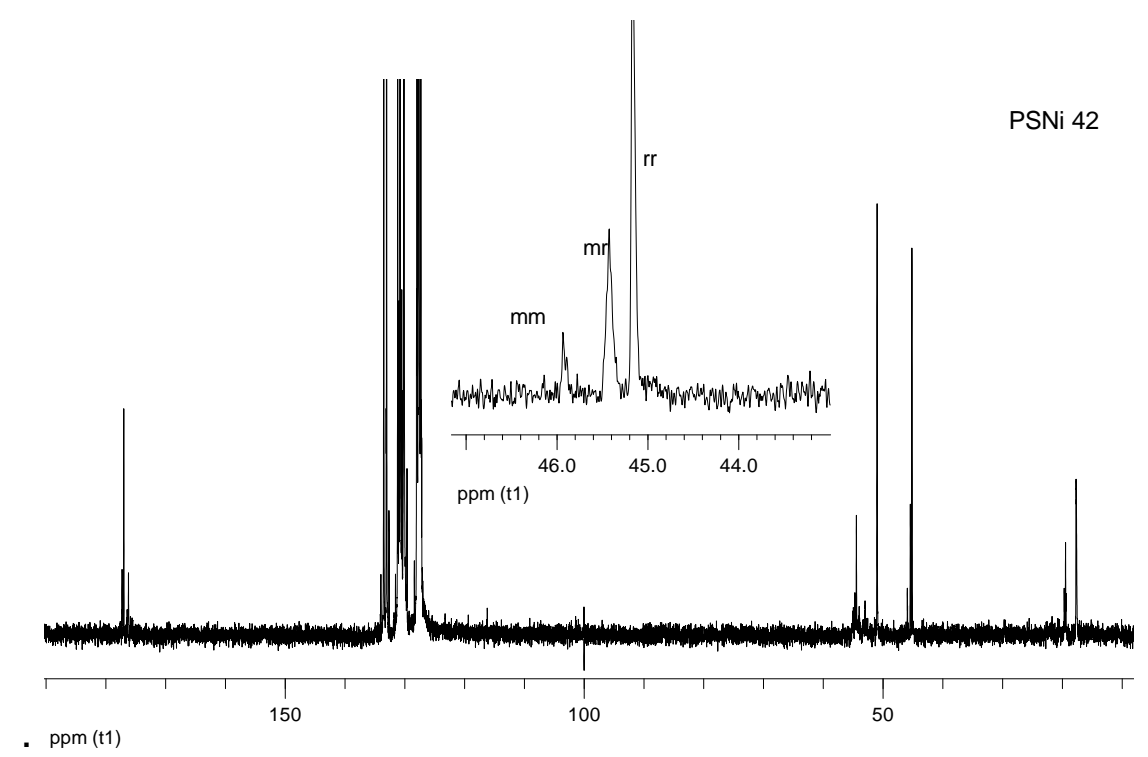

Fig. 4. ${ }^{13} \mathrm{C}$ NMR spectrum of PMMA polymer rich in syndiotacticity. Run PSNi42 (300 $\left.\mathrm{MHz}, \mathrm{TCB}, 110^{\circ} \mathrm{C}\right)$.

Tab. 10. Tacticity of PMMA polymers.

\begin{tabular}{c|c|c|c|c}
\hline \multirow{2}{*}{ Run } & \multirow{2}{*}{$\begin{array}{c}\text { Experimental } \\
\text { conditions }\end{array}$} & \multicolumn{3}{|c}{ Tacticity (\%) } \\
\cline { 3 - 5 } & & $\mathbf{m m}$ & $\mathbf{m r}$ & $\mathbf{r r}$ \\
\hline PSNi 41 & $1 / \mathrm{MCM} 41 / \mathrm{MAO} 30^{\circ} \mathrm{C}$ & 12.6 & 29.4 & 58.0 \\
PSNi 43 & $1 / \mathrm{MCM} 41 / \mathrm{MAO} 0^{\circ} \mathrm{C}$ & 11.5 & 16.5 & 72.0 \\
PSNi 42 & $2 / \mathrm{MCM} 41 / \mathrm{MAO} 30^{\circ} \mathrm{C}$ & 12.3 & 31.5 & 56.2 \\
PSNi 45 & $2 / \mathrm{MCM} 41 / \mathrm{MAO} 0^{\circ} \mathrm{C}$ & 8.7 & 29.2 & 62.1 \\
LNi 168 & $1 / \mathrm{MAO} 0^{\circ} \mathrm{C}$ & 3.9 & 24.4 & 71.7 \\
LNi 153 & $1 / \mathrm{MAO} 30^{\circ} \mathrm{C}$ & 11.4 & 34.3 & 54.3 \\
LNi 166 & $2 / \mathrm{MAO} 0^{\circ} \mathrm{C}$ & 14.7 & 30.5 & 54.8 \\
LNi 164 & $2 / \mathrm{MAO} 30^{\circ} \mathrm{C}$ & 12.2 & 29.1 & 58.7 \\
\hline
\end{tabular}

The amount of ethylene in the reaction product was obtained by using the ${ }^{1} \mathrm{H}$ NMR data. Applying the method reported by Matsugi et al. [34]. The values of MMA shown in Table 9 correspond to the ratio between the integration values for $\mathrm{O}-\mathrm{CH}_{3}$ protons of MMA against the value obtained for the $\mathrm{CH}_{2}$ protons of the ethylene. System 2/MAO although less active than 1/MAO, seems to be a better system for the 
polymerization of the two monomers. The products of these reactions were first washed with methanol and then extracted with boiling chloroform in order to remove all the soluble PMMA fraction. The NMR spectrum of the extracted polymer still presents the characteristic resonances of both polymers, PE and PMMA. However, due to the low solubility of these products and the very few connections between the $\mathrm{PE}$ and PMMA blocks expectable to be present in these copolymers no conclusive NMR characterization could be achieved. Thus, in spite of several experimental details pointing to the formation of block copolymers, the possibility of these reaction products being blends of PE and PMMA cannot be excluded.

The heterogeneous catalytic systems remain active in the polymerization of MMA although a decrease in the catalytic activity is observed. Yield values are quite similar for both systems. In the case of $2 / \mathrm{MCM} 41 / \mathrm{MAO}$ at $30^{\circ} \mathrm{C}$, the heterogeneous catalyst shows even a slightly higher yield when compared to the homogeneous one. However, with 1/MCM41/MAO a loss of around 3 times in yield is observed when compared to the homogeneous system

The polymethylmetacrylate obtained was analysed by ${ }^{1} \mathrm{H}$ and ${ }^{13} \mathrm{C} R M N$. The polymers obtained show some degree of syndiotacticity, regardless the catalytic system used. The use of both homogeneous and heterogeneous catalysts results in polymers with similar microstructure. The integration values of the 3 resonances at 44.5; 44.7 and 45.3 ppm assigned to the quaternary carbon were used for the tacticity determination

Figure 4 shows an example of a carbon spectrum of these polymers.

Syndiotacticity seems to be higher when polymerization is carried out at lower temperatures. Comparing the results obtained for the homogeneous and heterogeneous systems one can conclude that the microstructures of the polymers are not very different. The heterogeneization process only affects the catalytic activity and not the microstructure of the polymers.

\section{Conclusions}

The work presented in this paper showed that it is possible to prepare heterogeneous catalysts active in the polymerization of ethylene following the proposed methods.

The first method consisted in direct impregnation of the alpha diimine nickel complexes in the support and has shown to be a quite efficient one since the resulting heterogeneous catalysts have remained highly active. The polymer obtained with these heterogeneous catalysts presents the same structural characteristics as those obtained with the corresponding homogeneous systems. By using the right experimental conditions a polymer with very small catalyst contamination could be produced. The main disadvantage of this heterogeneization method is the lower content of organometallic complex in the support making it difficult to be properly characterized.

The second method presented in this paper also gave rise to active heterogeneous catalysts. The modification of the original metal zeolite catalyst with the introduction of the organic ligand resulted in an increase in the catalytic activity of the system. Even so, these catalysts were not as active as those obtained by the direct impregnation method. However, comparing the catalysts obtained by Method $A$ and Method B is somewhat difficult since they have very different porous structures and chemical characteristics. While MCM-41 has much larger pores than $\mathrm{Y}$ zeolite, the 
latter is able to bind cationic species in a very efficient way, due to the negative nature of the zeolite framework.

These catalysts were also able to homo- and copolymerize methylmethacrylate with ethylene, showing the polymers obtained some degree of syndiotacticity. Once again the presence of the carrier seems not to affect the microstructure of the synthesized polymers.

\section{Experimental part}

\section{Materials and general procedures}

All manipulations were carried out under an atmosphere of nitrogen using glove box and standard Schlenk techniques, unless stated otherwise. Complexes 1 and 2 and ligands L1, L2 and L3 were synthesized as described in the literature [34-36].

Solvents were pre-dried over activated $4 \AA$ molecular sieves or sodium wire (in the case of toluene) and then freshly distilled under an atmosphere of nitrogen from the drying agents. Trimethylaluminum (TMA), tri-i-butylaluminum (TIBA), and diethylaluminum chloride (DEAC) were all purchased from Aldrich and used without any further purification. Methylaluminoxane PMAO-IP with $7 \% \mathrm{Al}$ and $1.7 \%$ free TMA, was purchased from Akzo Chemical Company and used as received.

Ethylene (N25) was supplied by Air Liquide and passed over a bed of molecular sieves before being used in polymerization.

\section{Polymerization procedure}

Polymerizations of ethylene were carried out in a flame dried $250 \mathrm{ml}$ crown capped pressure bottle sealed with neoprene septum and pump filled with nitrogen atmosphere after three vacuum/nitrogen cycles. $50 \mathrm{ml}$ of dry toluene were introduced in this polymerization bottle and after evacuating the nitrogen, the reactor was saturated with ethylene at the desired pressure. This pressure was kept nearlyconstant throughout the polymerization reactions by using an on/off controller with a dead zone, which opens an inlet valve when the pressure falls below a predetermined threshold, and closes it again when it exceeds another preset value.

The co-catalyst was added in the proper $\mathrm{Al} / \mathrm{Ni}$ ratio via a glass syringe. At this time, the solution was allowed to equilibrate for 5 minutes. After this, the corresponding amount of a catalyst suspension in toluene was added to the polymerization reactors via a glass syringe equipped with a proper needle. The polymerization runs were terminated after the desired time by quenching the mixture with $150 \mathrm{ml}$ of a $1 \%$ $\mathrm{HCl} /$ methanol solution. The obtained polymers were then filtered, washed several times with $50 \mathrm{ml}$ portions of methanol and dried in a vacuum oven at $50{ }^{\circ} \mathrm{C}$ for three days.

\section{Catalyst preparation}

The organometalic compounds were synthesized according to the literature [35-37]. The heterogeneous catalysts were prepared by two different methods: by the direct impregnation of the complex in the support (Method A) and by the reaction of the ligand to the metal previously incorporated in the support (Method B). 
-Method A - direct impregnation

This method was used with MCM 41 as support material. The impregnation process consisted in contacting a solution of known concentration with the support for a certain period of time. After this contact the samples were washed several times.

To a suspension of $2 \mathrm{~g}$ of MCM41 in $40 \mathrm{ml}$ of dry toluene, a toluene solution of the complex $(15 \mathrm{ml})$ was added so that the total amount of complex would be around $5 \%$ $(\mathrm{w} / \mathrm{w})$ in relation to the support. The resulting suspension was heated at $65^{\circ} \mathrm{C}$ and stirred over night for $17 \mathrm{~h}$. At room temperature the suspension was filtered and the catalyst was washed consecutively with dry toluene in a total of $450 \mathrm{ml}$. The resulting powder was dried at reduced pressure (6 to $\left.9 \times 10^{-2} \mathrm{mbar}\right)$ for $16 \mathrm{~h}$.

For catalyst precursor 1, bis[N,N'-(4-tert-butyl-diphenylsilyl-2,6-diisopropylphenyl) imino]acenaphthene dibromonickel, a light orange powder was obtained, which will be designated as 1/MCM41. With Catalyst precursor 2, bis[N,N'-(4-tert-butyldiphenylsiloxy-2,6-dimethylphenyl)imino]acenaphthene\} dibromonickel, a light pink powder was obtained and it will be designated as 2/MCM41.

Tab. 11. Structure of the diimine compounds studied.

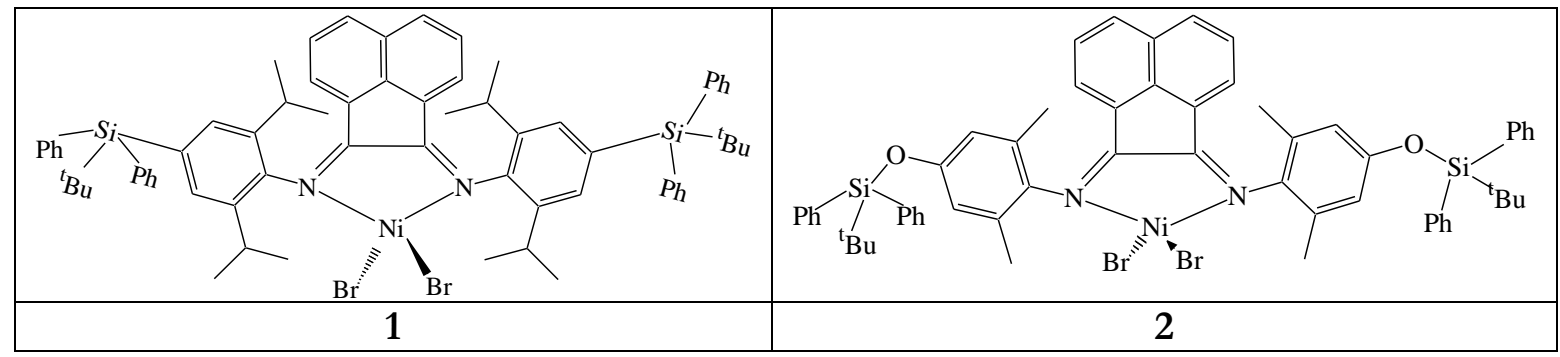

-Method B - Reaction with the ligand

Three different ligands L1, L2, e L3, prepared as described in the literature and shown in Figure 5, were used to synthesise the catalysts studied in this work.

All these compounds belong to Brookhart's ligands family and the substituents in the amine ring account for the differences in their structures. The main goal was to study the effect of the molecule size of the ligand in the heterogenization process and features of the catalyst.

For this synthesis, similar conditions to those reported in the literature for the homogeneous coordination reaction of the ligands to the metal, were used.

A concentrated solution of the ligand in $\mathrm{CH}_{2} \mathrm{Cl}_{2}$ was carefully added to $0.99 \mathrm{~g}$ of $\mathrm{NaY}$ with $1.7 \% \mathrm{Ni}$. The metal has been previously introduced in the zeolite by the ion exchange method. This solution was prepared by using only the necessary volume of solvent to dissolve the amount of ligand in a $1 / 1 \mathrm{~mol}$ ratio to the metal. The suspension was stirred for 7 days at room temperature. The solid was filtered and washed with solvent $(20 \mathrm{ml})$, several times to a total of $200 \mathrm{ml}$. The resulting powders were dried under reduced pressure for $16 \mathrm{~h}$. Catalysts referenced as L1/NiY, L2/NiY and L3/NiY were obtained.

Similar procedure was used for MCM41 impregnated by the incipient method with $1 \%$ of Ni. Catalysts L1/NiMC41 and L2/NiMC41 were thus obtained 


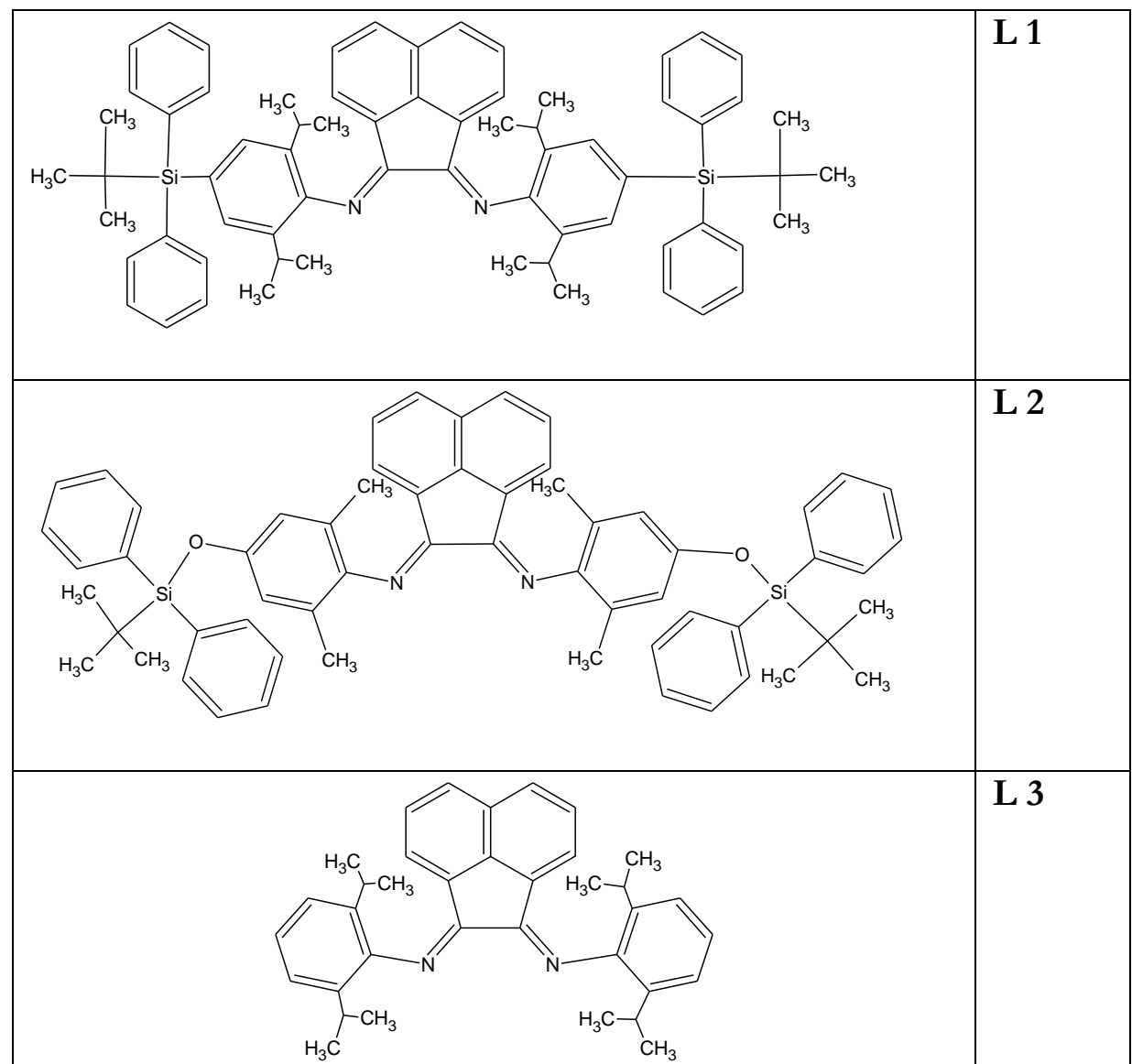

Fig. 5. Ligands used to prepare the heterogeneous catalysts

\section{Catalysts and polymers characterization}

Metal analyses were obtained from the Laboratório de Análises do IST by Inductively coupled Plasma - ICP

The NMR spectra were recorded on a Varian Unity $300\left({ }^{1} \mathrm{H}, 300 \mathrm{MHz} ;{ }^{13} \mathrm{C}, 75.43\right.$ $\mathrm{MHz}$ ) spectrometer and referenced internally using residual proton- $\left({ }^{1} \mathrm{H}\right)$ and carbonsolvent $\left({ }^{13} \mathrm{C}\right)$ resonances relative to tetramethylsilane (TMS, $\left.\delta=0 \mathrm{ppm}\right)$. NMR analyses of the polyethylenes and polymethylmethacrylate were performed on samples dissolved in a mixture of $1,2,4$-trichlorobenzene $/ \mathrm{C}_{6} \mathrm{D}_{6}(\mathrm{v} / \mathrm{v}=1: 1)$ at $110^{\circ} \mathrm{C}$, and the internal reference was provided by hexamethyldisiloxane $\left(\delta{ }^{1} \mathrm{H} 0.058, \delta{ }^{13} \mathrm{C}\right.$ 1.9 relative to TMS).

The thermal properties of the polymers were determined by Differential Scanning Calorimetry (DSC). The polymers were subjected to a heating ramp between $30^{\circ} \mathrm{C}$ and $200^{\circ} \mathrm{C}$ using a heating rate of $10^{\circ} \mathrm{C} / \mathrm{min}$ and a cooling rate less than $10^{\circ} \mathrm{C} / \mathrm{min}$ for DSC analysis. The amount of inorganic matter in the polymerization reaction product was determined by thermal gravimetric analysis (TGA). The equipment used for the DSC/TGA analyses was a SDT 2960 simultaneous DSC/TGA from TA Instruments.

Textural characterization of catalysts performed by nitrogen adsorption at $-196{ }^{\circ} \mathrm{C}$ was done in a micrometrics ASAP 2010.

UV-Vis DRS spectra of the solids was performed in a Varian Cary 5000 UV-VIS-NIR equipped with a special device Praying Mantis, Harrick, for reflectance mode. 
The XPS data was obtained with a XPS Kratos XSAM800 operated in the fixed analyser transmission mode using the Al $\mathrm{K}$ alpha-radiation.

\section{Acknowledgements}

This work was supported in part by Fundação para a Ciência e Tecnologia, and FERDER (Projects POCTI/32775/EQU/99 and POCTI/45104/EQU/2002). Inês Matos (BD/10338/2002 and SFRH/BPD/34659/2007) is grateful to FCT. We appreciate the support by Borealis in the polymerization equipment.

\section{References}

[1] Coates, G.C. Chem. Rev., 2000, 100 (4), 1223.

[2] Ittel, S.D.; Jonhson, L.K.; Brookhart, M. Chem. Rev. 2000, 100, 1169.

[3] Alt, H.; Koppl, A. Chem. Rev. 2000, 100, 1205.

[4] Svern, J.R.; Chadwick, J.C.; Duchateau, R.; Friederichs, N. Chem. Rev. 2005, 105, 4073.

[5] Welborn, H.C. U.S. Patent 4808 561, 1989. Chem. Abstr 1987, 106, 157033.

Takahashi, T. U.S. Patent 5026 797, 1991 Chem. Abstr. 1989, 111, 58539.

[6] Franceschini, F.C.; Travares, T.T.R.; dos Santos, J.H.Z.; Soares, J.B.P. Macromol. Chem. Phys. 2004, 205, 1525.

[7]Chu, K.-Y.; Soares, J.B.P.; Penlidis, A. J. Polym. Sci., Polym. Sci. 2000, 38, 462.

[8] Shan, C.L.P.; Chu, K.-J.; Soares, J.B.P.; Penlidis, A. Macromol. Chem. Phys. 2000, 201, 2195.

[9] Chao, C.; Pratchayawutthirat, W.; Praserthdam, P.; Shiono, T.; Rempel, G. L. Macromol. Rapid Commun. 2002, 23, 672.

[10] Jongsomjit, B.; Kaewkrajang, P.; Wanke, S.E.; Praserthdam, P. Catal. Lett. 2004, 94, 205.

[11] Guimaraes, R.; Stedile, F.C.; dos Santos, J.H.Z. J. Mol.Catal. A 2003, 206, 353.

[12] Galland, G.B.; Seferin, M.; Guimaraes, R.; Rohrmann, J.A.; Stedile, F.C.; dos Santos, J.H.Z. J. Mol. Catal. A 2002, 189, 233.

[13] Alonso, C.; Antinolo, A.; Carrillo-Hermosilla, F.; Carrion, P.; Otero, A.; Sancho, J.; Villasenor, E. J. Mol. Catal. A 2004, 220, 286.

[14] Arrowsmith, D.; Kaminsky, W.; Laban, A.; Weingarten, U.Macromol. Chem. Phys. 2001, 202, 2161

[15] Miller, C.J.; O'Hare, D. Chem. Commun 2004, 1710

[16] Hlatky, G.G. Chem. Rev. 2000, 100, 1347.

[17] Dupuy, J.; Broyer, J.-P.; Spitz, R.; Malinge, J. Macromol. Chem. Phys. 2001, 202, 2038.

[18] Lambert, J.-F. ; Hoogland, M. ; Che, M. J. Phys. Chem. 1997, 101, 10347.

[19] Boujday, S. ; Lambert, J.-F. ; Che, M. Chem. Phys. Chem. 2004, 5, 1003.

[20] Cavillot, V.; Champagne, B. Int. J. Quantum Chem. 2005, 101, 840.

[21] Lepetit, C.; Che, M. J. Phys. Chem. 1996, 100, 3137.

[22] Soibinet M., De'champs-Olivier I., Guillon E., Barbier J., Aplincourt M., Chuburu F., Baccon M., Handel H. Polyhedron 2005, 24, 143.

[23] Fernandes, S.; Soares, A.; Lemos,L.; Lemos, M.A.N.D.A.; Mano, J.F.; Maldanis, R.J.; Rausch, M.D.; Chien, J.C.W.; Marques, M.M. J. Organomet. Chem. 2005, 690, 895.

[24] Gates, D.P.; Svejda, S.A.; Oñate, E.; Killian, C.M.; Johnson, L.K.; White, P.S.; Brookhart, M. Macromolecules 2000, 33, 2320. 
[25] Chien, J. C. W.; Fernandes, S.; Correia, S. G.; Rausch, M. D.; Dickinson, L.C.; Marques,M. M., Polym Int 2002, 51, 729.

[26] Correia, S.G.; Marques, M.M.; Ascenso, J.R.; Ribeiro, A.F.G.; Gomes, P.T.; Dias, A.R.; Blais, M.; Rausch, M.D.; Chien, J.C.W. J. Polym. Sci. Part A: Polym. Chem. 1999, 37, 2471.

[27] Galland, G.B.; de Souza, R.F.; Mauler, R.S.; Nunes, F.F. Macromolecules 1999, 32, 1620.

[28] Johnson, L.K.; Killian, C.M.; Brookhart, M. J. Am. Chem. Soc.. 1995, 117, 6414.

[29] I. Matos, Y. Zhang, I Fonseca, F. Lemos, MANDA Lemos, F. Freire, A. C. Fernandes, Ana M. Botelho do Rego, A. Valente, J. F. Mano, R. T. Henriques, M.M. Marques, e-Polymers 2006, $n^{\circ}$ 075, http://www.e-polymers.org.

[30] Cameron, P. A.; Gibson, V.C.; Graham, A. J., Macromolecules 2000, 33, 4329.

[31] Collins, S.; Ward, D.G J. Am. Chem. Soc., 1992, 114, 5460.

[32] Endo, K.; Inukai, A., Polymer Internacional 2000, 49, 110.

[33] Kim, I.; Hwang, J.-M.; Lee, J. K.; Ha, C. S.; Woo, S.I. Macromol. Rapid Commun. 2003, 24, 508.

[34] Matsugi, T.; Kojoh, S.-I.; Kawahara, N.; Matsuo, S.; Kaneko, H.; Kashiwa, N., J. Polymer Science: Part A: Polymer Chemistry 2003, 41, 3965

[35] Liu, H.-R.; Costa, S.I.; Gomes, P.T.; Duarte, M.T.; Branquinho, R.; Fernandes, A.C.; Chien, J.C.W.; Marques, M.M. J. Organomet. Chem., 2005, 690 (5), 1314.

[36] Yuan, J.-C.; Silva, L.C.; Gomes, P.T.; Valerga, P.; Campos, J.M.; Ribeiro, M.R.; Chien, J.C.W.; Marques, M.M., Polymer 2005, 46, 2122.

[37] Maldanis, R.J.; Wood, J.S.; Chandrasekaram, A.; Rausch, M.D.; Chien, J.C.W. J.Organomet.Chem. 2002, 645, 158. 